\title{
Low-Pressure Induced Disproportionation of Barium Distannide
}

\author{
Amanda H. Trout ${ }^{1,2}$, Kelsey L. Hodge ${ }^{3}$, Mike Scudder ${ }^{3}$, Joshua E. Goldberger ${ }^{3}$, David W.
}

$\mathrm{McComb}^{1,2 *}$

${ }^{1}$ Center for Electron Microscopy and Analysis, The Ohio State University, Columbus, Ohio 43210, USA;

${ }^{2}$ Department of Materials Science and Engineering, The Ohio State University, Columbus, Ohio 43210, USA.

${ }^{3}$ Department of Chemistry and Biochemistry, The Ohio State University, $151 \mathrm{~W}$ Woodruff Ave, Columbus, 43210

Ohio, USA;

\section{Removal of the zero-loss peak}

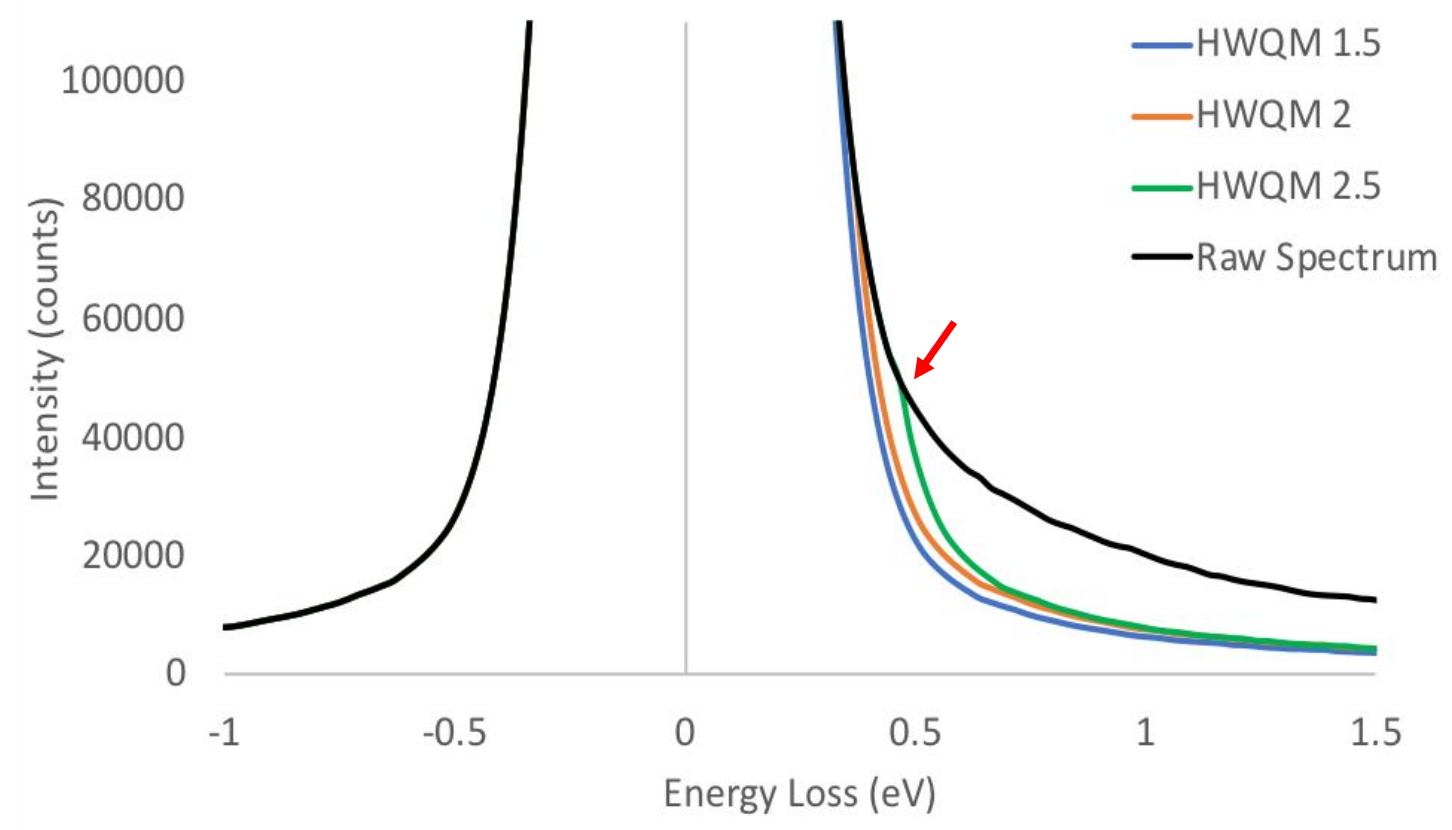

Figure S1. Raw spectrum (black) and the ZLP models with varying HWQM (blue, orange, green). A HWQM of 2 (orange) was chosen as the HWQM of 2.5 (green) causes a kink in the spectrum indicated by the red arrow. 


\section{Variation of the refractive index for Kramers-Kronig analysis}

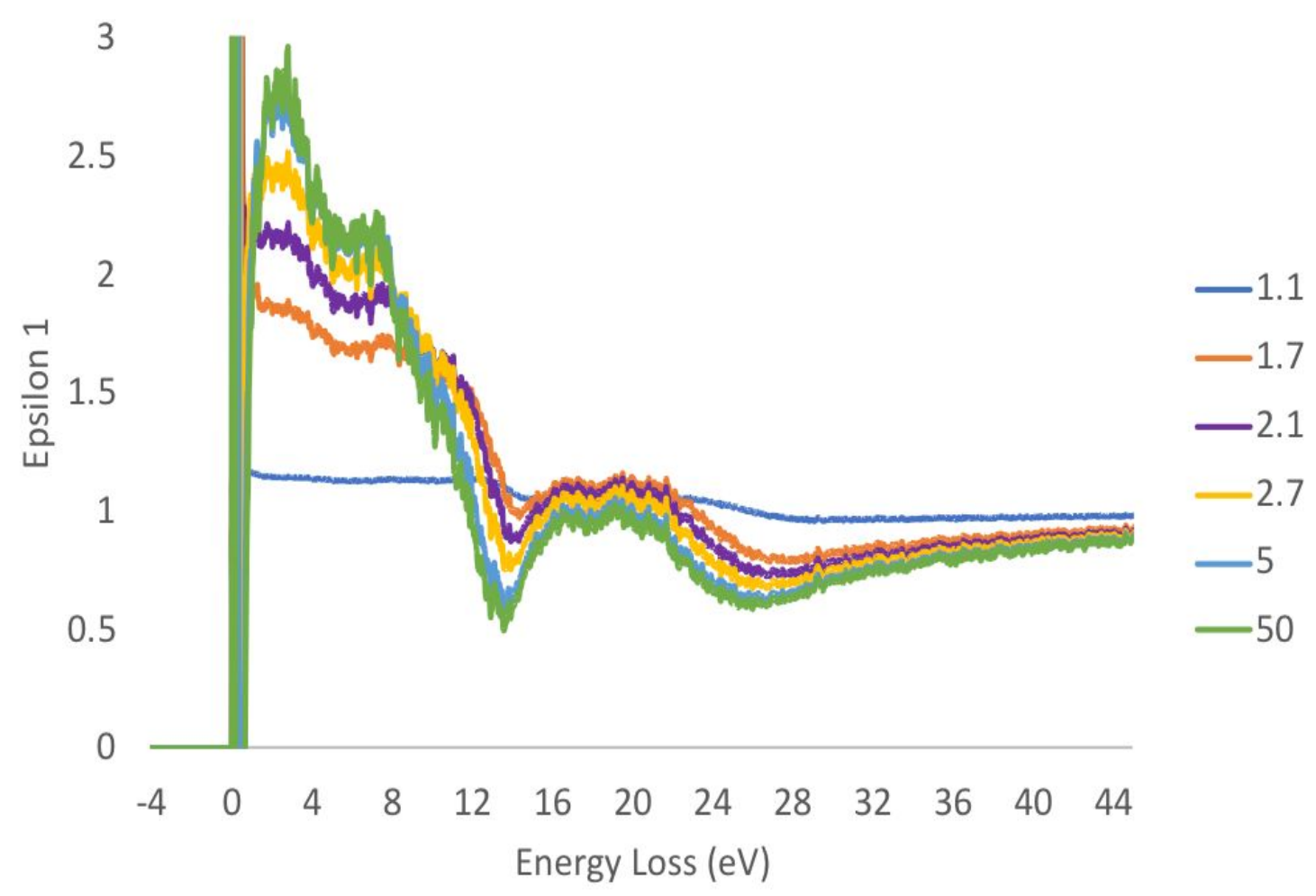

Figure S2. Plot of epsilon 1 for different refractive index values. A refractive index of 2.7 (yellow) was chosen for all Kramers-Kronig analyses as this value is close to the refractive index of Sn. 


\section{Powder XRD of BaSn $n_{2}$ left in the glovebox}

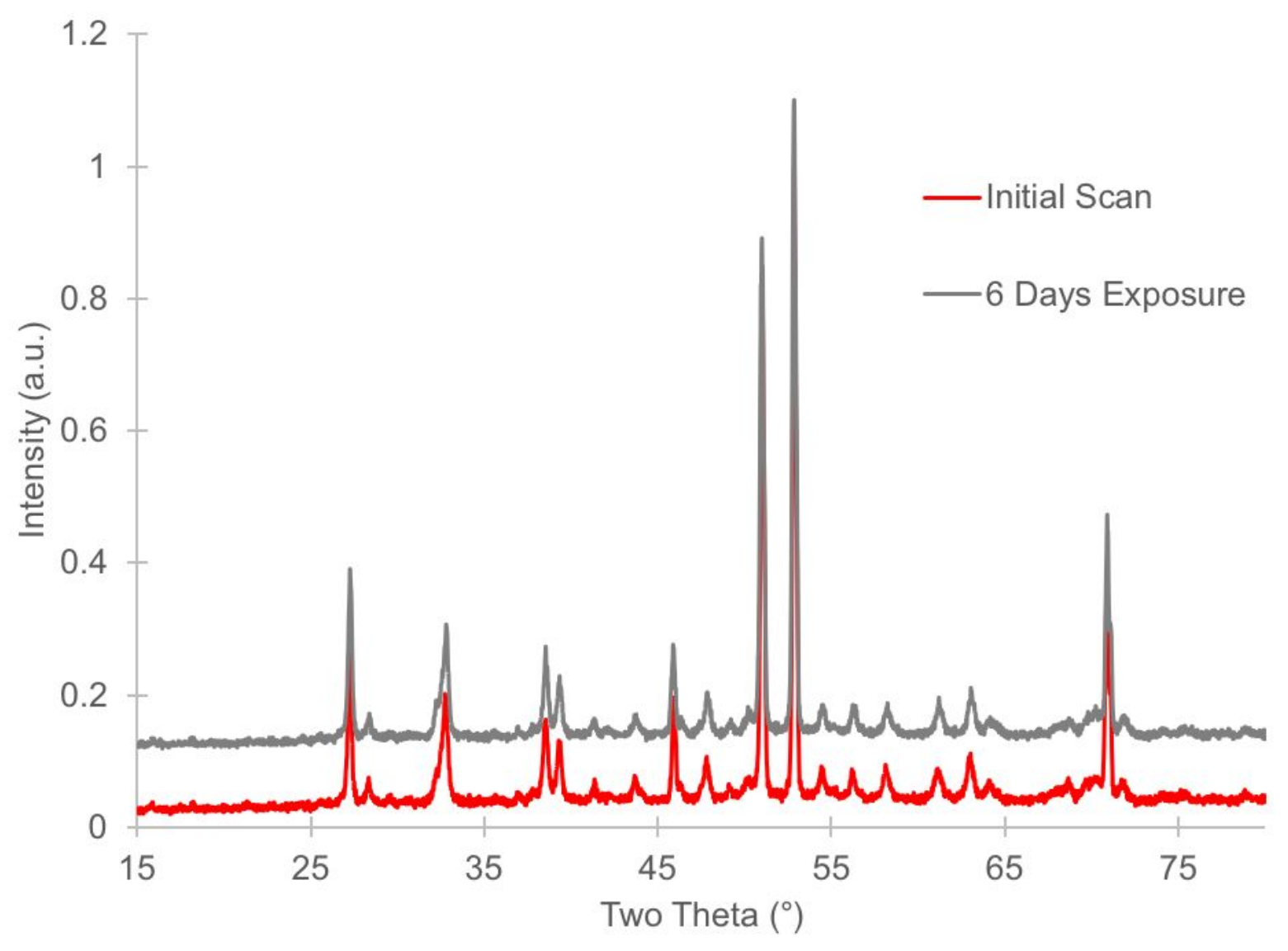

Figure S3. Powder XRD of $\mathrm{BaSn}_{2}$ ground in a glovebox and left exposed in the glovebox for 6 days. The before (red) and after (gray) are superimposable with no $\beta$-Sn observed indicating that trace amounts of oxygen exposure is not enough to drive disproportionation. 